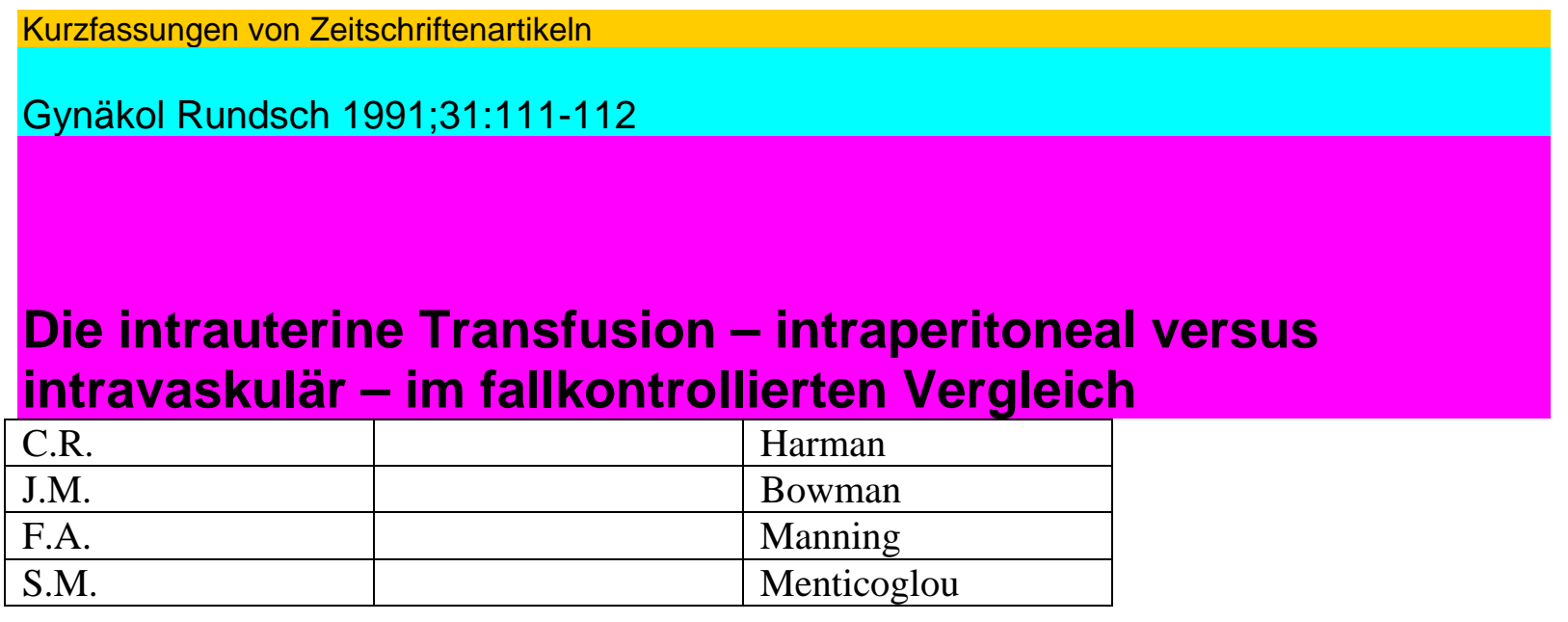

Woman's Hospital, Winnipeg, Man., Canada

Die Methode der intraperitonealen fetalen Transfusion zur Behandlung schwerer fetaler Alloimmunerkrankungen ist weit verbreitet und wird seit 25 Jahren angewendet. Es besteht jedoch seit einigen Jahren die Alternative der intravaskulären fetalen Transfusion, welche derzeit nur an einigen wenigen Zentren durchgefiihrt wird. Standen bisher zur Evaluierung des Managements vor dem Eingriff nur Fruchtwasserbestimmungen und Ultraschall zur Verfügung, so beinhaltet die intravaskuläre fetale Transfusion einen Gefásszugang und damit die Verfügbarkeit hämatologischer und biochemischer Daten sowie die Aussage über den respiratorischen Säure-Basen-Haushalt.

Das Prozedere ist technisch angenehmer: Eine dünne Nadel wird mit hoher Sicher-heit in das präzis definierte Ziel gebracht, während anderseits bei einer intraperitonealen Transfusion, mit einem Unsicherheitsfaktor behaftet, mittels einer grossen Nadel die fetale Peritonealhòhle punktiert wird. Weiter ware noch der Unterschied in den Ergebnis-sen beider Methoden bei schweren Erkrankungen (moribunder Hydrops fetalis) zu bemer-ken. Durch die fehlende fetale Atembewegung eines moribunden Feten, welche essentiell für die Absorption des Transfusionsproduktes einer intraperitonealen fetalen Transfusion ist, sind die Chancen auf Erfolg sehr gering, im Gegensatz zu jenen bei der intravaskulären fetalen Transfusion. Zwischen Juni 1980 und Mai 1986 wurden 75 Feten der Therapie einer intraperitonealen fetalen Transfusion unterzogen. Der Transfusionszeitpunkt basierte auf Standard-kriterien in der Anamnese, Fruchtwasseranalyse und fetalen Ultraschalluntersuchung. Die Zweittransfusion wurde im Abstand von 7 bis 10 Tagen durchgefiihrt. Eventuelle Wiederholungstransfusionen wurden im 28-Tage-Intervall durchgefiihrt. Mittels Ultra-schalls wurde das fetale Wohlbefinden kontrolliert. Ab Mai 1986 wurden 44 Feten mittels ultraschallgeleiteten intravaskulären fetalen Transfusionen behandelt, bei welchen insge-samt 174 Transfusionen durchgefiihrt wurden (3,9 pro Fetus). Im Gegensatz dazu wurden in der Gruppe mit der intraperitonealen fetalen Transfusion 104 Transfusionen (2,4 pro Fetus) durchgefiihrt. Die intravaskuläre Methode zeigte eine höhere Erfolgsrate bei der Ersttransfusion im Gegensatz zu der intraperitonealen ( $p=0,016$, Student-t-Test). Im weiteren Vergleich von intravaskulärer zu intraperitonealer fetaler Transfusion zeigte sich, dass bei ersterer eine spätere Gestationswoche (34,1 vs. 30,7; p = 0,011), eine höhere Rate der vaginalen Entbindung (34 vs. 17; p = 0.05), erhöhte Hämoglobinwerte zum Zeitpunkt der Geburt (117 gm/1 vs. 107 gm/1; ns), eine geringere Anzahl von Austauschtransfusionen ( 0,8 vs. 1,$8 ; p=0,007)$ sowie ein kürzerer Aufenthalt auf der Intensivstation 


\section{Kurzfassungen von Zeitschriftenartikeln}

\section{2}

(6,1 vs. 8.2 Tage; $p$ = 0,044) möglich waren. Die Überlebensrate von 40/44 (91\%) mit intravaskulärer fetaler Transfusion im Vergleich zu 29/44 (66\%) mit intraperitonealer fetaler Transfusion zeigt einen signifikanten Unterschied $(p=0,005)$.

Für die Klinik: Die Methode der intravaskulären fetalen Transfusion findet immer weitere Verbreitung in der Behandlung schwerer alloimmunologischer Erkrankungen und verdrängt die derzeit in vielen Zentren angewendete Methode der intraperitonealen fetalen Transfusion. Diese Studie vergleicht beide Methoden in einem fallkontrollierten Design. Die intravaskuläre Methode zeigt in alien Punkten bessere Ergebnisse, was das Überleben, die Gestationswoche und eine niedrigere Komplikationsrate der Mutter betrifft. Die intraperitoneale fetale Transfusionsmethode ist als eine Second-line-Me-thode bei speziellen Fallen anzusehen. G. Heytmanek, Wien Aus: Am J Obstet Gynecol 1990;162:1099-1103 cit. Gynäkol Rundsch 1991;31:112-113 Onkogene Transformation durch humane Papillomaviren-Typ-16-DNS in Anwesenheit von Progesteron oder Progestin aus oralen Kontrazeptiva Oncogenic Transformation by Human Papillomavirus Type 16 Deoxyribonucleic Acid in the Presence of Progesterone or Progestins from Oral Contraceptives

Alan Pater, Mahin Bayatpour, Mary M. Pater

Faculty of Medicine, Health Science Center, Memorial University of

Newfoundland, St. Johns, Newfoundland, Canada, and Fresno, Calif., USA

Das Plattenepifhelkarzinom der Cervix uteri ist das zweithäufigste Karzinom der Frau. Beobachtungen deuten auf eine sexuell bedingte Ätiologie hin. In diesem Zusammen-hang wurde die Rolle der humanen Papillomaviren (HPV) bei flachen Kondylomen der Zervix dokumentiert. Histologische Studien zeigten, dass die HPV-bedingten Läsionen oft zusammen mit Dysplasien, Carcinomata in situ sowie invasiven Karzinomen auftreten. Studien mit Hilfe von DNS-Hybridisationstechniken und molekularen Klonierungsverfah-ren bewiesen das Vorhandensein und die Freisetzung von HPV-16- und -18-DNS bei 70-80\% der Zervixkarzinome und zervikalen Tumorzellinien. Zusätzlich zu den spezifischen HPV-DNS sind andere Substanzen, wie Hormone oder Nikotin, als Kofaktoren für die Entstehung einer zervikalen Neoplasie von Bedeutung. Andere Studien zeigten, dass bei Verwendung oraler Kontrazeptiva ein grösseres Risiko für die Entstehung zervikaler Neo-plasien besteht. Auch hormonale Veränderungen in der Schwangerschaft können zu einer höheren Anfálligkeit für HPV-bedingte Infektionen im unteren Genitaltrakt führen.

In dieser Arbeit wird der Beweis für eine onkogene Zelltransformation durch Kom-bination von HPV-16-DNS und dem humanen raí-Onkogen bei Zusatz von Progesteron oder dem chemisch verwandten Progestin, Norgestrel, der pharmakologisch wirksamen Substanz oraler Kontrazeptiva, geführt. Keine onkogene Zelltransformation durch HPV-16-DNS und dem rasOnkogen wurde unter Östrogeneinwirkung erreicht. Intrauterine Transfusion - Intraperitoneal versus Intravascular Approach:

a Case-Control Comparison 